\title{
Operation, Calibration and Performance of the CMS Silicon Tracker
}

\section{Steven Lowette ${ }^{* \dagger}$, for the CMS Collaboration}

University of California, Santa Barbara, USA

E-mail: steven.lowettedcern.ch

The CMS tracker is the largest silicon detector ever built. It consists of the inner pixel detector, with 66 million silicon pixels, surrounded by the strip tracker with almost 10 million readout channels and an active area of close to $200 \mathrm{~m}^{2}$. These detectors, with an operational fraction of over 98\%, have been calibrated and aligned both with cosmic rays and LHC pp collisions at 0.9 , 2.4, and $7 \mathrm{TeV}$ centre-of-mass energy. The excellent performance of the CMS tracker with LHC collisions is described, showing the results of precise commissioning, efficient operations and continuous monitoring of the CMS tracker.

35th International Conference of High Energy Physics - ICHEP2010,

July 22-28, 2010

Paris France

* Speaker.

${ }^{\dagger}$ The work of S.L. is supported by the U.S. Department of Energy, under contract No. DE-FG02-91ER40618 


\section{The CMS tracker}

The Compact Muon Solenoid (CMS) detector [1], one of two multipurpose experiments at the Large Hadron Collider (LHC), is built around a superconducting coil which provides an axial magnetic field of $3.8 \mathrm{~T}$. The CMS tracker is fully contained within this solenoid. It is designed to provide a precise and efficient measurement of charged particle trajectories and of the primary and secondary vertices they originate from. The many environmental and physics constraints led to a design based entirely on silicon detector technology. Closest to the collision point the pixel detector is found, composed of three barrel layers (BPIX) and two endcap disks (FPIX), the innermost layer at a radius of $4.3 \mathrm{~cm}$. The $\sim 1.1 \mathrm{~m}^{2}$ of active sensor area is read out by 66 million electronic channels. Around the pixel detector the silicon strips are placed in 10 barrel layers and 3 plus 9 endcap disks, arranged in four subsystems: the inner barrel (TIB), the outer barrel (TOB), the inner disks (TID) and the endcaps (TEC). Almost $200 \mathrm{~m}^{2}$ of sensor area is readout over 9.3 million electronic channels. All in all the CMS tracker provides highly efficient tracking up to a pseudorapidity of 2.5. A schematic overview of the tracker design is shown in Figure 1.

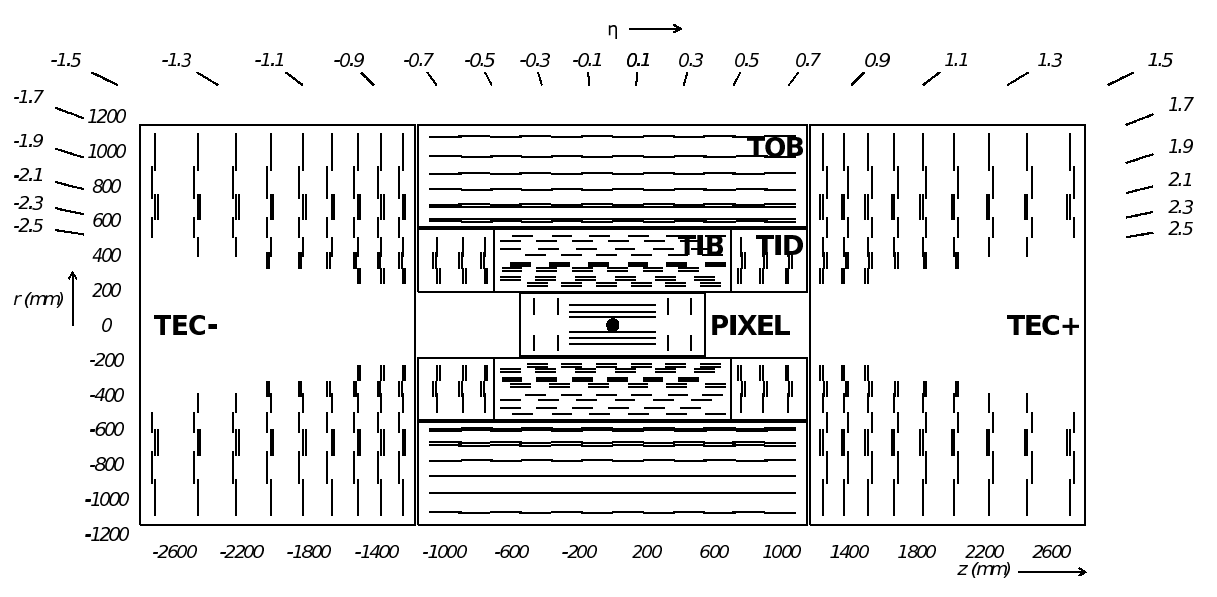

Figure 1: Schematic overview of the CMS tracker design.

\section{Detector commissioning}

The readout chips of the strip tracker can operate in two modes. In peak mode, used throughout 2009, a single sample is read out at the maximum of the signal pulse. While this mode has a low noise and is robust against time misalignment, the so-called deconvolution mode needs to be used to read out the tracker during LHC operations with short bunch spacing, This requires ns precision on the internal synchronization of each detector module, along with pulse shape tuning. Other calibration steps during strip commissioning include tuning of the lasers for the optical readout links, tuning of the analog baseline and the measurement of the pedestal and noise for each individual channel.

The pixel detector, just like the strip tracker, is also readout over optical links for every Level-1 hardware trigger accept, with off-detector digitization. In contrast to the strips, though, the pixel data is zero suppressed in the on-detector readout chip, with an adjustable threshold per pixel. In 
terms of commissioning the pixel readout chain needs similar calibrations as the strips, like the laser settings and the timing including fine delay adjustments using collisions. Pixel specific are the response calibration pixel-by-pixel and the zero suppression threshold optimization.

The calibration of the tracker has profited extensively from the hundreds of millions of cosmic rays recorded in dedicated runs in 2008 and 2009 [2]. It allowed for the validation and finetuning of the above mentioned calibrations, but also the performance measurements, calibrations and alignment detailed in the subsequent sections $[3,4,5]$. As a result the CMS tracker registered the first LHC collisions as a remarkably well prepared detector.

\section{Tracker performance with collisions}

The measurement of deposited charge for high-quality tracks is one of the basic performance measurements. For the strips the figure of merit is the signal-to-noise ratio $(\mathrm{S} / \mathrm{N})$, which both in peak mode ( $900 \mathrm{GeV}$ collisions) and in deconvolution mode ( $7 \mathrm{TeV}$ collisions) agrees well with the expectation [6]. Deconvolution readout indeed exhibits a larger noise, leading to an $\mathrm{S} / \mathrm{N}$ of about 20 throughout the detector, as shown in Figure 2. For the pixels the cluster charge is scaled by the track path length and the sensor thickness. A very good data-simulation agreement is observed in both barrel and endcaps, as shown in Figure 3. This agreement validates the pixel readout calibration.

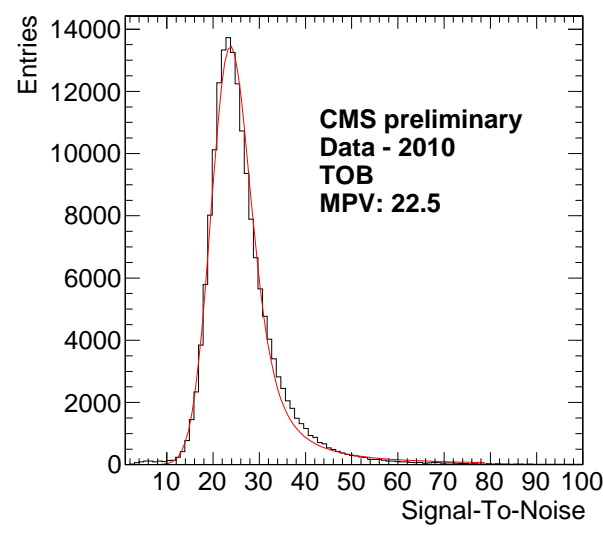

Figure 2: Signal-to-noise ratio in deconvolution mode for strips in the outer barrel.

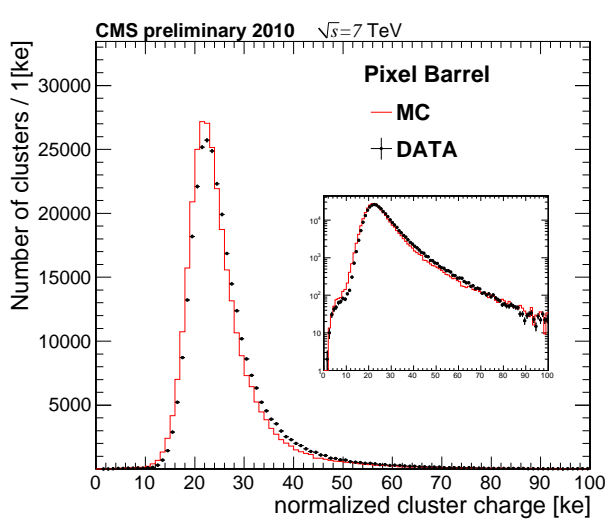

Figure 3: Normalized cluster charge in the barrel pixels.

A next step in the performance validation is the measurement of the hit reconstruction efficiency. The used technique checks for a hit where one is expected from hits in neighbouring layers. This module-by-module efficiency determination allowed to spot several problems on individual modules. When excluding known problems a hit reconstruction efficiency of over $99.9 \%$ is measured in the strips, while in the pixels also an efficiency of well over $99 \%$ is observed, which is well modeled by the simulation.

For the measurement of the strip and pixel spatial hit resolution overlapping modules in the barrel are used. To minimize the effect of multiple scattering, misalignment or track extrapolation, this resolution is extracted from the residual of the double differential of hit and track position. For the strips the measurement with cosmic rays with negligible track angle yields a resolution 
of $17.2 \pm 1.9 \mu \mathrm{m}$ in the inner layers up to $23.2 \pm 3.6 \mu \mathrm{m}$ in the outer layers, in agreement with the simulation. Also for the pixels good agreement is obtained, with a measured resolution from collisions of $12.7 \pm 2.3 \mu \mathrm{m}$ and $28.2 \pm 1.9 \mu \mathrm{m}$ in respecitvely the $x$ and $y$ direction.

An important correction to cluster position estimates is needed because of the Lorentz force on the drifting charges in the sensors. This has a direct impact on the tracker alignment. By measuring the minimum of the cluster width versus the incident track angle the so-called Lorentz angle can be derived. With cosmic rays the tangent of this angle was determined to be $0.409 \pm 0.001$ (BPIX, Figure 4), $0.081 \pm 0.005$ (FPIX), $0.007 \pm 0.002$ (TIB) and $0.09 \pm 0.01$ (TOB), where all uncertainties are statistical only. In collisions the BPIX value was confirmed with the cluster width and an alternative method. In the case of the strips an additional correction needs to be accounted for in the case of the deconvolution mode, where a fraction of the charge doesn't reach the sensor backplane in time for the readout.

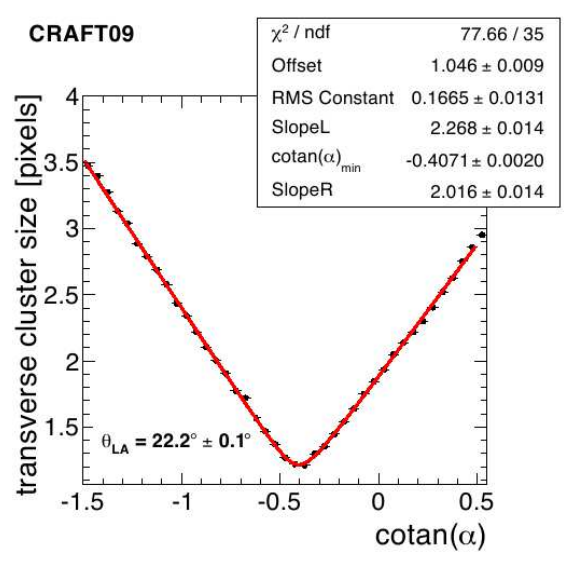

Figure 4: Lorentz angle in the barrel pixel from the minimization of the cluster width.

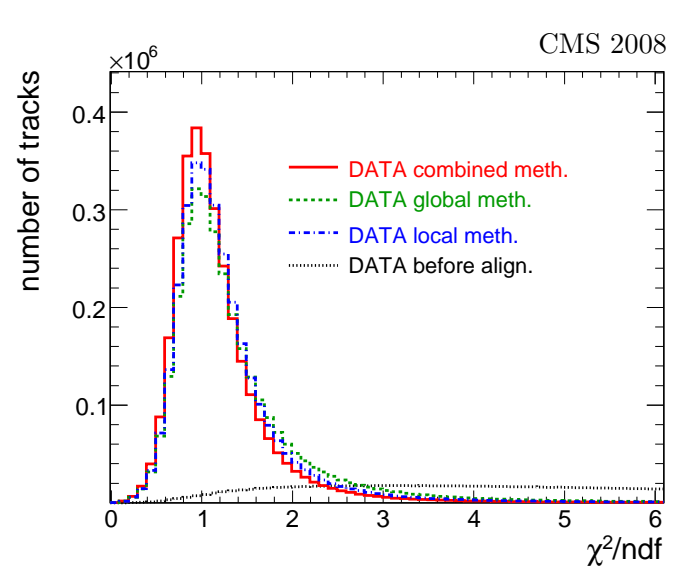

Figure 5: Improvement of track $\chi^{2}$ values by the individual and combined methods with cosmic data.

What concerns alignment, two complementary track-based algorithms are used. A global method minimizes residuals with only a few iterations by applying a matrix size reduction without loss of correlations or precision. The other method searches a local uncorrelated solution for each module. A large number of iterations is needed for this method in the case of a large misalignment. The final results are obtained by running both methods in sequence. A first alignment campaign was performed with cosmic rays prior to collisions, with best results in the barrel. An update with collisions using tracks from minimum-bias collisions brought further improvement, mostly in the forward region. In Figure 5 the improvement of track $\chi^{2}$ values by the individual and combined methods is shown with cosmic data.

A final demonstration of the performance of the CMS tracker comes from the particle identification capabilities. First, all strip readout channels were calibrated to a uniform energy response. With this input it is then possible to contruct the energy loss distribution as a function of the particle momentum, as shown in Figure 6. At high $\mathrm{d} E / \mathrm{d} x$ kaons, protons and deuterons can be distinguished. Doing this by selecting $\mathrm{d} E / \mathrm{d} x>5 \mathrm{MeV} / \mathrm{cm}$ the particle masses can be estimated, as shown in Figure 7. The comparison with the simulation shows an excellent agreement for kaons and protons, but reveals as well the absence of deuterons in the simulation. 


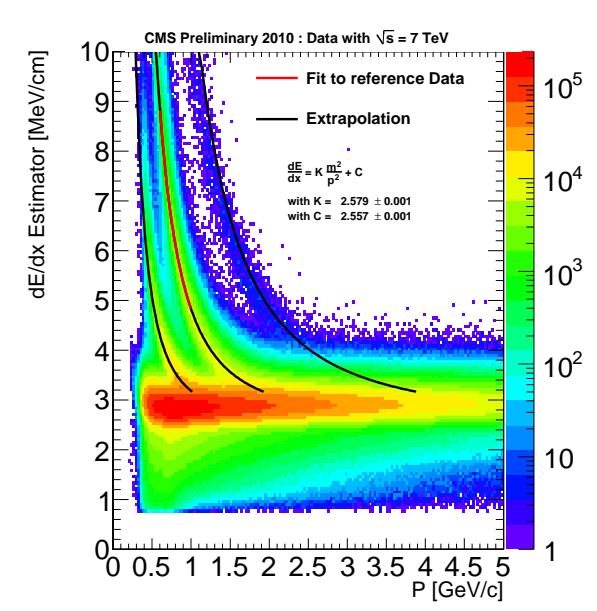

Figure 6: Energy loss $\mathrm{d} E / \mathrm{d} x$ in the strips as a function of the particle momentum.

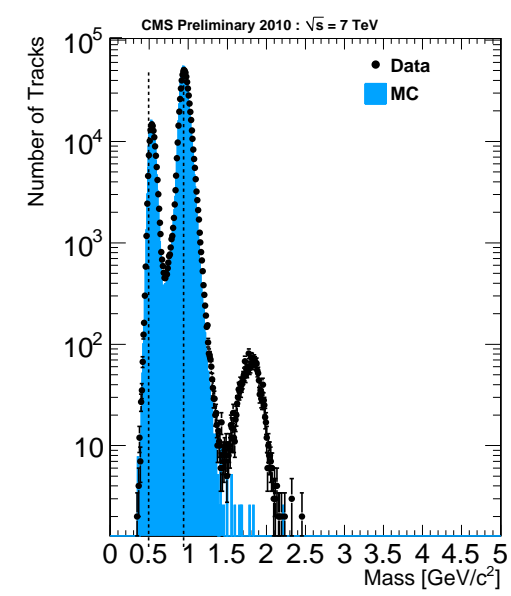

Figure 7: Mass estimate in data and simulation for particles with $\mathrm{d} E / \mathrm{d} x>5 \mathrm{MeV} / \mathrm{cm}$.

Several of the above performance measurements have now become routine calibration tasks, automatically run promptly on the incoming data. A cornerstone of the efficient recording of the data with the CMS tracker comes from fast-feedback as well as long-term monitoring of the detector environment and the data quality. An essential tool for this is the Data Quality Monitoring infrastructure, which monitors the detector and reconstruction performance as well online for fast feedback as offline for the more detailed data certification.

\section{Conclusions}

The CMS tracker, the largest silicon tracking detector ever built, has been recording very efficiently high-quality data during the first pp LHC collision runs. This bears proof of a remarkably well-prepared detector with more than $98 \%$ operational fraction. With collisions the efficient tracker operations and excellent performance were confirmed, and collision data is continuously used to further improve calibrations and alignment.

\section{References}

[1] CMS Collaboration, "The CMS experiment at the CERN LHC," JINST 3, S08004 (2008).

[2] CMS Collaboration, "Commissioning of the CMS Experiment and the Cosmic Run at Four Tesla," JINST 5, T03001 (2010) [arXiv:0911.4845 [physics.ins-det]].

[3] CMS Collaboration, "Commissioning and Performance of the CMS Pixel Tracker with Cosmic Ray Muons,” JINST 5, T03007 (2010) [arXiv:0911.5434 [physics.ins-det]].

[4] CMS Collaboration, "Commissioning and Performance of the CMS Silicon Strip Tracker with Cosmic Ray Muons,” JINST 5, T03008 (2010) [arXiv:0911.4996 [physics.ins-det]].

[5] CMS Collaboration, "Alignment of the CMS Silicon Tracker during Commissioning with Cosmic Rays,” JINST 5, T03009 (2010) [arXiv:0910.2505 [physics.ins-det]].

[6] CMS Collaboration, "CMS Tracking Performance Results from Early LHC Operation,” arXiv:1007.1988 [physics.ins-det]. 